\title{
Evaluación de la actividad antimicrobiana de los extractos de la planta Lippia origanoides h.b.k. Cultivada en el departamento del Quindío
}

\section{Evaluation of antimicrobial activity of Lippia origanoides h.b.k. Plant extracts cultivated in the department of Quindio}

\author{
Julieth Henao*, Leidy J. Muñoz**, Eunice Ríos V.***, \\ Leonardo Padilla****, Germán Antonio Giraldo G. *****
}

Recibido:Septiembre 28 de 2009

Aceptado: Diciembre 1 de 2009

Correspondencia: Laboratorio de Investigación Agroindustria de Frutas Tropicales, Universidad del Quindío Avenida Bolivar Calle 12 norte Armenia Quindío. Email:erios@uniquindio.edu.co

\section{RESUMEN}

Se realizó el estudio fitoquímico preliminar de las hojas de Lippia origanoides H.B.K., llevado a cabo por varios métodos de extracción (percolación, soxhlet y lixiviación), utilizando solventes de diferentes polaridades como éter de petróleo, hexano, diclorometano y etanol. Se analizó el contenido de metabolitos secundarios, encontrando en los extractos polares flavonoides, taninos, glicósidos cardiotónicos, hidroquinonas y alcaloides, y en los extractos apolares, carotenoides, glicósidos cardiotónicos y esteroles. La actividad antimicrobiana de los extractos polares y apolares se evaluó por el método de difusión en disco (Kirby-Bauer) modificado, utilizando bacterias Gram-negativas y Gram-positivas, hongos y levaduras, observando que los extractos apolares mostraron actividad de inhibición del crecimiento de los microorganismos evaluados, mientras que los extractos polares no mostraron actividad frente a los mismos microorganismos.

Palabras clave: estudio fitoquímico, Lippia origanoides, actividad antimicrobiana.

\begin{abstract}
A preliminary phytochemical study was conducted with leaves of Lippia origanoides H.B.K., carried out by several extraction methods (percolation, Soxhlet and leaching), using solvents of different polarities such as petroleum ether, hexane, dichloromethane, and ethanol. The content of secondary metabolites was analyzed found in polar extracts flavonoides, tannins, cardiac glycosides, hydroquinone and alkaloids, and in the nonpolar extracts, carotenoids, cardiac glycosides and sterols. The antimicrobial activity of polar and nonpolar extracts was evaluated by the disk diffusion method (Kirby-Bauer) modified, using Gram-negative and Gram-positive bacteria, fungi and yeast, noting that the nonpolar extracts showed activity of grown inhibition of the microorganism test, whereas the polar extracts did not show activity to the same microorganisms. Key words: phytochemical study, Lippia origanoides, antimicrobial activity.
\end{abstract}

\section{INTRODUCCIÓN}

Las plantas han desempeñado un papel fundamental en la vida del hombre, quien las ha utilizado para suplir necesidades básicas como alimento, medicina, vivienda y vestido, incluso en actos rituales. El uso de las plantas es una práctica que existe desde los inicios de la especie humana [1].
Los productos naturales, principalmente los de origen vegetal han sido la principal fuente de agentes terapéuticos de la humanidad durante siglos, constituyendo su uso una costumbre profundamente arraigada en las culturas de los pueblos. En los últimos 20 años ha habido un resurgimiento de la investigación de productos naturales, debido a la gran diversidad química y biológica del reino vegetal, la cual es una

\footnotetext{
* Laboratorio de Investigación Agroindustria de Frutas Tropicales, Programa de Química, Universidad del Quindío.

** Laboratorio de Investigación Agroindustria de Frutas Tropicales, Programa de Química, Universidad del Quindío.

***Grupo de Investigación Agroindustria de frutas tropicales, Programa de Química, Universidad del Quindío.

**** Grupo de Inmunología Molecular (GYMOL). Universidad del Quindío.

***** Grupo de Investigación Agroindustria de frutas tropicales, Programa Ingenieria de Alimentos, Universidad del Quindío.
} 
fuente rica y un recurso renovable para el desarrollo de nuevas moléculas de interés farmacéutico. Se estima que más del 90 por ciento de las especies vegetales no han sido estudiadas para el descubrimiento y desarrollo de nuevas moléculas de interés farmacéutico. Los productos naturales representan el $50 \%$ de las drogas de uso clínico en países en vías de desarrollo [2].

Los estudios de plantas en el área de los productos naturales, aspiran establecer relaciones entre la composición química de sus metabolitos secundarios y sus propiedades farmacológicas.

Además, el conocimiento de las propiedades químicas de los aceites esenciales y otros extractos, permiten proponer a estos productos como fuente alternativa y natural, de compuestos útiles en la industria y tecnología (cosmética, alimentos, fragancias, síntesis química).

El género Lippia se caracteriza por reunir especies a las que se les atribuyen cualidades aromáticas.

Sin embargo, aún hay plantas dentro de este género que no han sido lo suficientemente estudiadas para ser incluidas, por su utilidad, en este campo de aplicación. Es el caso de Lippia origanoides H.B.K., un arbusto silvestre en el nordeste de Suramérica y algunos países de Centroamérica y las Antillas. Lippia origanoides H.B.K. alcanza $3 \mathrm{~m}$ de longitud, posee hojas verdes ovadas muy aromáticas e inflorescencias en racimo, axilares y blancas.

Generalmente esta planta se conoce como "Orégano", las infusiones de sus flores y hojas, son consumidas para tratar afecciones respiratorias e intestinales, diarreas y disentería y tiene un gran interés fitoterapéutico, pues tradicionalmente se ha utilizado como antiséptico [3].

Variaciones en la composición química de metabolitos secundarios en plantas de la misma especie, ha conducido a designar quimiotipos, esto permite apuntar a variaciones genéticas en la especie $[4,5,6]$.

\section{MATERIALES Y MÉTODOS}

\section{Material vegetal}

Las hojas de L. origanoides, en estado de floración, fueron donadas por la Asociación Bioagroindustrial, ASOBAl, y se recolectaron en dos jornadas, 6:00 a.m. y 4:00 p.m., en la finca El Faro de la Suiza, vereda el Plataniyal, corregimiento de Pueblo Tapao, Municipio de Montenegro, ubicado sobre 1282 m.s.n.m. y con una temperatura promedio de $27^{\circ} \mathrm{C}$.
Determinación del contenido de humedad del Material vegetal

La humedad de las hojas de la planta recolectada en ambas jornadas se determinó siguiendo el método A.O.A.C. 20.013/19801.0, con la siguiente ecuación:

$$
\% \text { humedad }=\frac{M_{i}-M_{f}}{M_{i}} * 100
$$

Donde: Mi: masa inicial

Mf: masa final

\section{Obtención de los extractos por Soxhlet y lixiviación}

El material vegetal se sometió a extracción por Soxhlet utilizando éter de petróleo como solvente y el marco se utilizó para la obtención del extracto polar, sometiéndolo a lixiviación con etanol absoluto durante 8 días.

\section{Obtención de extractos por percolación en frío}

Se realizaron extracciones sucesivas utilizando solventes de diferentes polaridades como hexano, diclorometano y etanol absoluto, obteniendo así tres extractos, los cuales se concentraron hasta la tercera parte a presión reducida por medio de un rotavapor.

\section{Pruebas fitoquímicas preliminares}

Se realizaron pruebas fitoquímicas a cada uno de los extractos obtenidos para evidenciar presencia de: taninos, flavonoides, hidroquinonas, carotenoides, esteroles, glicósidos cardiotónicos, saponinas, coumarinas y alcaloides $[7,8]$.

\section{Actividad antimicrobiana}

La actividad antimicrobiana de los extractos de Lippia origanoides H.B.K. se realizó siguiendo la técnica de difusión en disco de Kirby - Bauer. Los microorganismos evaluados fueron las bacterias Gram-negativas Escherichia coli, Pseudomonas aeruginosa, Aeromona hydrophila, Proteus mirabilis, Enterobacter cloacae, Klebsiella Pneumoniae, las bacterias Gram-positivas Staphylococcus aureus y Enterococcus gallinarum y la levadura Candida albicans, utilizando el agar Mueller Hinton como medio de crecimiento para todos los microorganismos. El control positivo utilizado fue Amikacina $(30 \mu \mathrm{g})$ y el control negativo fue el solvente respectivo de cada extracto. Las placas se incubaron a $37^{\circ} \mathrm{C}$ por $24 \mathrm{~h}$, y después de este tiempo se midió el diámetro de la zona de inhibición ( $\mathrm{mm}$ ).

\section{RESULTADOS Y DISCUSIÓN}

Determinación del contenido de humedad del material vegetal 
Tabla 1. Porcentaje de humedad de las hojas de Lippia origanoides recolectadas en dos jornadas.

\begin{tabular}{|c|c|}
\hline $\begin{array}{l}\text { Jornada de } \\
\text { recolección }\end{array}$ & $\begin{array}{c}\% \\
\text { Humedad }\end{array}$ \\
\hline 6: 00 a.m. & 58.3241 \\
\hline 4: 00 p.m. & 66.2264 \\
\hline
\end{tabular}

De acuerdo con la tabla 1, se encontró que el contenido de humedad de las hojas de Lippia origanoides H.B.K. es mayor para la jornada de recolección de las 4:00 p.m., lo cual implica que las horas de la mañana son las más apropiadas para el corte, ya que los cúmulos de agua de la planta se han deshidratado durante la noche, es decir, que al haber condiciones de oscuridad, la planta efectúa un proceso de reposo de su actividad fotosintética y devuelve al suelo el agua que tomó en el día [9], esto facilita la extracción de los metabolitos secundarios.

Pruebas fitoquímicas preliminares de los extractos obtenidos por Soxhlet y lixiviación

Los metabolitos secundarios fueron identificados de manera cualitativa mediante ensayos de coloración y precipitación en cada uno de los extractos polares y apolares de las hojas de la planta recolectadas en ambas jornadas. Los resultados de las pruebas se encuentran en la Tabla 2. en donde se utilizan las siguientes convenciones para la abundancia del metabolito en el extracto:

$\begin{array}{lc}\text { *Presentes en gran cantidad } & +++ \\ \text { *Presentes en mediana cantidad } & ++ \\ \text { *Presentes en pequeña cantidad } & + \\ \text { *Ausentes } & -\end{array}$

Tabla 2. Metabolitos secundarios de los extractos obtenidos por Soxhlet y lixiviación.

\begin{tabular}{|l|c|c|}
\hline Pruebas & $\begin{array}{l}\text { Extracto } \\
\text { etanólic o }\end{array}$ & $\begin{array}{l}\text { Extracto } \\
\text { eté reo }\end{array}$ \\
\hline Flavonoides & +++ & - \\
\hline Taninos & ++ & - \\
\hline Hidroquinonas & +++ & - \\
\hline Esteroles & - & +++ \\
\hline Saponinas & - & - \\
\hline Cardiotónicos & ++ & +++ \\
\hline Coumarinas & - & - \\
\hline Alcaloides & ++ & - \\
\hline Carotenoides & - & +++ \\
\hline
\end{tabular}

En el extracto etanólico de las hojas de Lippia origanoides se encontró la presencia de flavonoides con anillo de $\gamma$ benzopirona, taninos derivados del ácido protocatéquico, hidroquinonas, glicósidos cardiotónicos y alcaloides.

En el extracto etéreo se encontraron esteroles, glicósidos cardiotónicos y carotenoides.

Pruebas fitoquímicas preliminares de los extractos obtenidos por percolación

Los metabolitos secundarios fueron identificados de manera cualitativa mediante ensayos de coloración y precipitación en cada uno de los extractos de la planta obteniendo los resultados mostrados en la Tabla 3.

Tabla 3. Metabolitos secundarios de los extractos obtenidos por percolación.

\begin{tabular}{|l|c|c|c|}
\hline Prue bas & Ext. H & Ext. D & Ext. E \\
\hline Flavonoides & - & - & +++ \\
\hline Taninos & - & - & +++ \\
\hline Hidroquinonas & - & - & +++ \\
\hline Esteroles & +++ & +++ & - \\
\hline Saponinas & - & - & - \\
\hline Cardiotónicos & ++ & +++ & + \\
\hline Coumarinas & - & - & - \\
\hline Alcaloides & - & - & ++ \\
\hline Carotenoides & +++ & +++ & - \\
\hline
\end{tabular}

H: Hexano; D: Diclorometano; E: Etanol

En el extracto hexano se encontraron esteroles, glicósidos cardiotónicos y carotenoides.

En el extracto con diclorometano se observó la presencia de los mismos metabolitos hallados en el extracto hexano (glicósidos cardiotónicos, esteroles y carotenoides).

Mientras que en el extracto etanólico, al igual que el obtenido por lixiviación, se encontraron flavonoides, taninos, hidroquinonas, y alcaloides, y glicósidos cardiotónicos.

Al comparar el extracto etéreo con el de hexano, ambos muy apolares, se encuentra que la presencia de metabolitos es la misma, por lo tanto, se puede decir que el método de extracción (Soxhlet o Percolación) no tiene influencia sobre la composición del extracto, porque se esperaba que por Soxhlet, por efectos de la utilización de temperatura, algunos metabolitos se podían degradar o volatilizarse fácilmente. De esta forma, para obtener el extracto apolar de un material vegetal, el método por el cual se extraiga es 
indiferente, pues ambas técnicas son eficientes y seguras. Igualmente esto sucedió con los extractos etanólicos obtenidos por dos métodos (lixiviación y percolación), donde no hubo diferencia en la composición de metabolitos secundarios.

La presencia de flavonoides y taninos, así como de alcaloides se ha reportado antes en un estudio realizado, atribuyéndole a estos metabolitos funciones de defensa contra insectos, resistencia a parásitos, de allí la gran importancia que presentan desde el punto de vista fitoquímico, con respecto a su amplia acción biológica [10].

Actividad antimicrobiana de los extractos obtenidos por Soxhlet y lixiviación

Al hacer una comparación de la actividad antimicrobiana con respecto a la polaridad del extracto (Tabla 4), se observó que la actividad del extracto polar (etanol) fue escasa, mientras que el extracto apolar (éter de petróleo) resultó efectivo contra todos los microorganismos evaluados.

Tabla 4. Diámetros de los halos de inhibición de los extractos obtenidos por Soxhlet y lixiviación.

\begin{tabular}{|c|c|c|c|}
\hline Microorganismos & & $\begin{array}{l}\text { ietro } \\
\text { halos } \\
\text { ción }\end{array}$ & $\begin{array}{l}\text { de } \\
\text { de } \\
\mathrm{mm} \text { ) }\end{array}$ \\
\hline \multirow{4}{*}{$\begin{array}{l}\text { Candida albicans } \\
\text { Enterococcus } \\
\text { gallinarum } \\
\text { Staphylococcus } \\
\text { aureus }\end{array}$} & EP & ET & AK \\
\hline & $\mathrm{N}$ & 12 & ---- \\
\hline & 7 & 14 & 20 \\
\hline & $\mathrm{N}$ & 13 & $\begin{array}{l}--- \\
\end{array}$ \\
\hline \multirow{4}{*}{$\begin{array}{l}\text { Aeromona hydrophila } \\
\text { Enterobacter cloacae } \\
\text { Escherichia coli } \\
\text { Klebsiella } \\
\text { pneumoniae }\end{array}$} & $\mathrm{N}$ & 14 & 17 \\
\hline & $\mathrm{N}$ & 16 & 17 \\
\hline & $\mathrm{N}$ & 30 & 20 \\
\hline & $\mathrm{N}$ & 12 & 19 \\
\hline \multirow{2}{*}{$\begin{array}{l}\text { Proteus mirabilis } \\
\text { Pseudomona } \\
\text { aeruginosa }\end{array}$} & $\mathrm{N}$ & 11 & 20 \\
\hline & $\mathrm{N}$ & 20 & 22 \\
\hline
\end{tabular}

N: Negativo, EP: extracto Etéreo., ET: extracto Etanólico.,

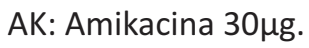

A)

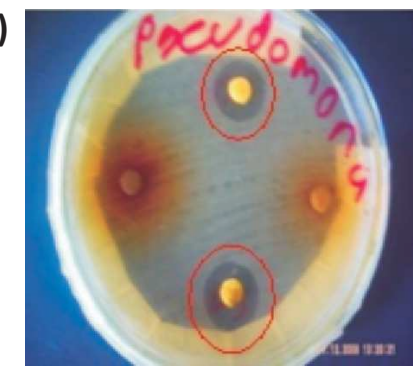

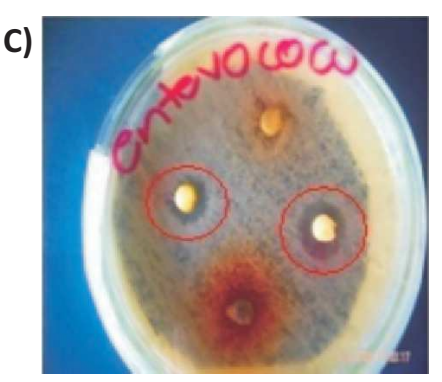

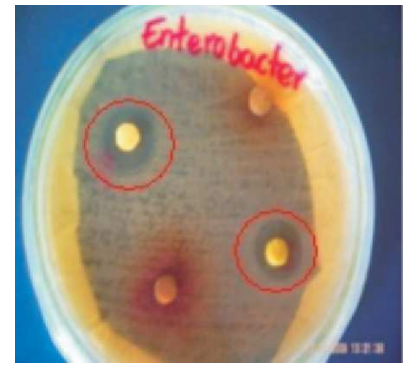

B)

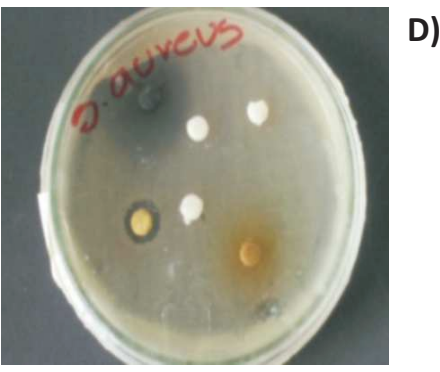

Figura 1. Halos de inhibición de los extractos etanólico y etéreo. Los del extractos etéreos están señalados con círculos rojos; a) actividad contra Pseudomonas

aeruginosa, con tamaños de 20 y $16 \mathrm{~mm}$ respectivamente);

b) actividad contra Enterobacter cloacae, diámetros del mismo tamaño (16mm); c) actividad contra Enterococcus gallinarum, 15 y $14 \mathrm{~mm}$ respectivamente; d) los discos blancos son los controles negativos con los solventes respectivos, no presentan actividad.

\section{Actividad antimicrobiana de los extractos de la percolación}

Al igual que con el extracto obtenido por lixiviación, la actividad del extracto de etanol no fue muy buena, pues solo inhibió el crecimiento de tres microorganismos. Sin embargo, los extractos de diclorometano de hexano mostraron una buena actividad frente a todos los microorganismos, aunque un poco mayor en el extracto de diclorometano (Tabla 5), indicando que los metabolitos responsables de dicha actividad tienen una polaridad intermedia.

En este extracto se puede evidenciar, además, una mayor actividad en las bacterias Gram-positivas debido, en gran parte, a la elevada resistencia a los antimicrobianos que poseen las Gram-negativas, y por la morfología de la membrana bacteriana.

Por el método de percolación los extractos mostraron una actividad ligeramente mayor que con aquellos obtenidos por el método de Soxhlet, indicando que, aunque el sometimiento del material vegetal a la acción de la temperatura no influye en la composición de metabolitos secundarios (al menos cualitativamente), puede disminuir en cierta forma su actividad antimicrobiana. 
Tabla 5. Diámetros de los halos de inhibición de los extractos obtenidos por percolación.

\begin{tabular}{|l|l|l|l|l|}
\hline \multirow{2}{*}{ M icroorganismo } & \multicolumn{4}{|l|}{$\begin{array}{l}\text { Diáme tros de Ios } \\
\text { halos de in hibición } \\
\text { (m m) }\end{array}$} \\
\cline { 2 - 5 } & EH & ED & EE & AK \\
\hline Candida albicans & 21 & 25 & $\mathrm{~N}$ & --- \\
\hline $\begin{array}{l}\text { Enterococcus } \\
\text { gallinarum }\end{array}$ & 20 & 22 & $\mathrm{~N}$ & 20 \\
\hline $\begin{array}{l}\text { Staphylococcus } \\
\text { aureus }\end{array}$ & 11 & 28 & $\mathrm{~N}$ & --- \\
\hline $\begin{array}{l}\text { Aeromona } \\
\text { hydrophila }\end{array}$ & 9 & 14 & $\mathrm{~N}$ & 17 \\
\hline $\begin{array}{l}\text { Enterobacter } \\
\text { cloacae }\end{array}$ & 7 & 21 & $\mathrm{~N}$ & 17 \\
\hline Escherichia coli & 15 & 21 & 8 & 20 \\
\hline $\begin{array}{l}\text { Kleb siella } \\
\text { pneumoniae }\end{array}$ & 11 & 13 & 12 & 19 \\
\hline Proteus mirabilis & 19 & 22 & 10 & 20 \\
\hline $\begin{array}{l}\text { Pseudomona } \\
\text { aeruginosa }\end{array}$ & 12 & 22 & $\mathrm{~N}$ & 22 \\
\hline
\end{tabular}

$\mathrm{N}$ : Negativo; EH: Extracto Hexano; ED: Extracto Diclorometano; EE: Extracto Etanol; AK: Amikacina $30 \mu \mathrm{g}$

\section{CONCLUSIONES}

La humedad de las hojas de Lippia origanoides fue mayor para la recolección de las 4:00 p.m., por lo tanto, el horario sugerido de corte es el de las 6:00 a.m. por su bajo contenido de agua, lo que permite una mejor extracción de cualquiera de sus componentes.

Se encontró también en los extractos polares obtenidos de las hojas de la planta flavonoides, quinonas, taninos, glicósidos cardiotónicos y alcaloides, y en su fracción apolar, esteroles, carotenoides y glicósidos cardiotónicos, sin que se presenten variaciones en la presencia de los metabolitos por la recolección del material vegetal en las dos jornadas diferentes, 6:00 a.m. y 4:00 p.m.

El extracto etéreo (apolar) obtenido por Soxhlet y los extractos de hexano y diclorometano (apolar y medianamente polar, respectivamente) obtenidos por percolación poseen actividad antimicrobiana contra $\mathrm{S}$. aureus, C. albicans, Pseudomonas aeruginosa, Aeromona hidrófila, E. coli, Proteus mirabilis, Enterococcus gallinarum, Klebsiella pneumoniae y Enterobacter cloacae.

La actividad antimicrobiana, fue mayor en levaduras y bacterias Gram-positivas que en bacterias Gram-negativas.

\section{AGRADECIMIENTOS}

Al programa de Química de la Universidad del Quindío y al laboratorio de investigación Diseño de Nuevos Productos DNP, también agradecemos a la Asociación Bioagroindustrial, ASOBAl, por la donación del material vegetal.

\section{BIBLIOGRAFÍA}

(1) Celis, A., Mendoza, C., Pachón, M., Cardona, José., Delgado, W., Cuca, L.E. Extractos Vegetales Utilizados como Biocontroladores con Énfasis en la Familia Piperaceae. Una revisión. Revista de Agronomía Colombiana. 2008; 26(1): 97106.

(2) Ortiz, G.H. Actividad Antifúngica de los Extractos Etanólicos de la Flor de Bourreria huanita y la Hoja de Lippia graveolens y sus Particiones Hexánica, Clorofórmica, Acetato de Etilo y Acuosa Contra los Hongos Sporothrix schenckii y Fonsecaea pedrosoi. Informe de Tesis presentado para optar el título de Química Bióloga. Universidad de San Carlos de Guatemala. 2006.

(3) Acevedo, A.M., Castañeda, M.L., Blanco, K., Cárdenas, C.Y., Reyes, J.A., Kouznetsov, V., Stashenko, E. Composición y Actividad Antioxidante de Especies Aromáticas y Medicinales con Alto Contenido de Timol y Carvacrol. Scientia et Technica. 2007; XIII(33): 125-128.

(4) Ruiz, C., Tunarosa, F., Martínez, J. \& Stashenko, E. Estudio Comparativo por GC-MS de Metabolitos Secundarios Volátiles de dos Quimiotipos de Lippia origanoides H.B.K., Obtenidos por Diferentes Técnicas de Extracción. Scientia et Technica. 2007; 8(33): 325-328.

(5) Pascual, M., Slowing, K., Carretero, E., Sánchez, D., y Villar A. Lippia: traditional uses, chemistry and pharmacology: a review. Journal of Ethnopharmacology. 2000; (76): 201-214. 
(6) Méndez, R., Serrano, J., Chataing, B., Jiménez, D., Mora, D., Rojas, L., Usubillaga, A. \& O`Callaghan, J. Estudio Comparativo de la Actividad Biológica del Aceite Esencial Protium heptaphylum (Aubl.) March y el Aceite Esencial Lippia origanoides H.B.K. Sobre Tres Especies de Nocardia sp. Instituto de Investigación, Facultad de Farmacia, Universidad de los Andes. 2007; 2(6): 49-52.

(7) Bilbao R., M. del R. Análisis Fitoquímico Preliminar. Edit. Universidad del Quindío. Armenia. 2007.

(8) Domínguez, X.A. Métodos de Investigación Fitoquímica. Centro Regional de Ayuda Técnica. AID. México. 1978.

(9) Rojas, R. Preservación de la Guadua. Universidad San Buenaventura-Cali. 2003.

(10)Rodríguez, D.A. \& Sanabria, M.E. Efecto del Extracto de Tres Plantas Silvestres sobre la Rizoctoniosis, la Mancha Sureña del Maíz y los Patógenos que la Causan. Revista Interciencia. 2005; 30(12): 739-744. 